Article

\title{
Active Shielding Design and Optimization of a Wireless Power Transfer (WPT) System for Automotive
}

\author{
Silvano Cruciani ${ }^{1}$, Tommaso Campi ${ }^{2, *}$, Francesca Maradei ${ }^{1}$ and Mauro Feliziani ${ }^{2}$ \\ 1 Department of Electrical Engineering and Automation, Sapienza University of Rome, 00185 Rome, Italy; \\ silvano.cruciani@gmail.com (S.C.); francesca.maradei@uniroma1.it (F.M.) \\ 2 Department of Industrial and Information Engineering and Economics, University of L'Aquila, \\ 67100 L'Aquila, Italy; mauro.feliziani@univaq.it \\ * Correspondence: tommaso.campi@univaq.it; Tel.: +39-086-243-4421
}

Received: 16 September 2020; Accepted: 23 October 2020; Published: 25 October 2020

check for updates

\begin{abstract}
This study deals with the optimization of a shielding structure composed by multiple active coils for mitigating the magnetic field in an automotive wireless power transfer (WPT) system at $85 \mathrm{kHz}$. Each active coil is independently powered and the most suitable excitation is obtained by an optimization procedure based on the Gradient Descent algorithm. The proposed procedure is described and applied to shield the magnetic field beside an electric vehicle (EV) equipped with SAE standard coils, during wireless charging. The obtained results show that the magnetic field in the most critical area is significantly reduced (i.e., approximately halved) with a very limited influence on the electrical performances (i.e., WPT efficiency decreases by less than 1 percentage point compared to the case without active shielding).
\end{abstract}

Keywords: active coil; automotive; electric vehicle (EV); electromagnetic compatibility; magnetic field; near field; shielding; wireless power transfer (WPT)

\section{Introduction}

Electric vehicles (EVs) are a key factor in the development of a more sustainable transport. Efficient and comfortable delivery of the electric power from the grid to the EV is crucial. For automotive applications, the most widely used technology for contactless charging is the wireless power transfer (WPT) technology based on magnetic resonant coupling as it permits transferring high power with an efficiency similar to that of plug-in charging [1-7]. When using this technology, an important problem is given by the magnetic field in the environment produced by the currents flowing in the coils of the WPT system [8-18]. Therefore, electromagnetic field (EMF) safety in terms of human exposure to electromagnetic fields needs to be addressed.

A typical WPT system for the wireless charging of an EV is composed by a transmitting coil $(T x)$ mounted on the ground and a receiving coil $(R x)$ mounted on the EV underbody. The resonance frequency is set to $85 \mathrm{kHz}$. The goal of the proposed work is the minimization of the magnetic flux density $\mathrm{B}$ in a zone outside the vehicle in close proximity to the $R x$ and $T x$ coils where people can stay. According to the definition in the SAE J2954 standard [7], there are three distinct regions to be considered, as shown in Figure 1. The most critical zone is Region 1 (under the chassis of the EV). However, being inaccessible to the humans, it does not cause any EMF safety problem. Region 3 is the zone inside the cabin occupied by the driver and the passengers where the magnetic field levels are not very high due to the shielding action of the metal bodyshell, at least in traditional cars made of steel or aluminum. The remaining zone is Region 2, i.e., beside and above the vehicle. The region 
beside the vehicle nearby the coils is the most critical in terms of risk for magnetic field exposure since the magnetic field is maximum there and could exceed the EMF safety limits [19-21].

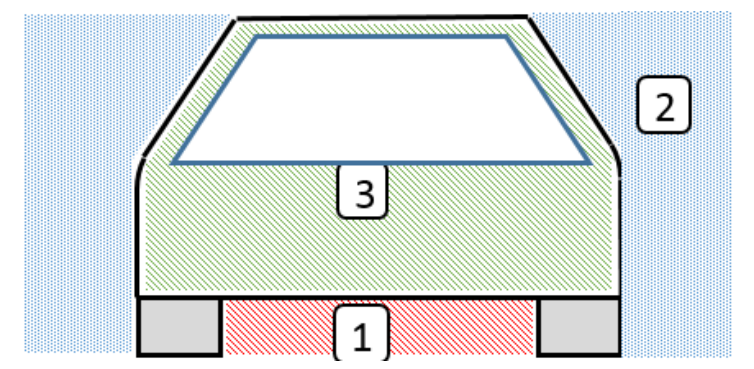

SAE regions:

1. Under the vehicle

2. Beside the vehicle

3. Inside the vehicle

Figure 1. SAE J2954 regions definition.

Being intentional sources of magnetic field, the WPT coils cannot be completely shielded as this would lead to a significant decrease of the system electrical performance [14]. In order to reduce the magnetic field, different shielding techniques can be used. The simplest one is based on the use of passive shields made of conductive materials. Despite the very good shielding performance obtained by these materials at the frequencies considered, eddy currents in the shield drastically affect the performance of a WPT system based on inductive technology, resulting in a significant reduction in efficiency [8]. On the other side, the use of magnetic materials with high permeability and negligible conductivity permits obtaining good shielding without affecting the electrical performances of the system. The main disadvantages of this solution are the weight and the cost of magnetic materials. The combined use of conductive and magnetic shields allows good shielding and electrical performances, but with an increase in weight and cost. Another well-known passive shielding technique is based on reactive coils, i.e., passive loops that are excited by time-varying magnetic fields produced by the currents induced by the source. Despite the great simplicity, the shielding performances of reactive coils are limited. A detailed description of this technique can be found in [16-18]. To overcome the mentioned inconveniences, active shielding is proposed here as a cost-effective solution for mitigating relatively low frequency electromagnetic fields, very suitable for automotive applications due the low impact on the weight.

The active shielding technique is definitely challenging [22-24], and consists in powering ad hoc designed active coils to generate a magnetic field opposite to the incident one in order to mitigate the total field. This technique is very efficient in reducing the static magnetic field, e.g., earth's magnetic field in medical applications, or to magnetically confine plasma in nuclear fusion devices. Active shielding techniques have been also applied to mitigate low frequency magnetic fields using a single active coil as in power line applications [22]. When a single active coil is not enough to obtain a satisfactory field reduction, it is possible to use a more complex configuration based on multiple active coils. In this case, the main issue is the definition of the electro-geometrical configuration of each coil (i.e., shape, dimension, orientation, and position), as well as the current to be injected. In the recent past, a procedure to mitigate the magnetic field at a single point has been proposed using an active coil configuration [23]. Here, this procedure is extended to a multicoil configuration. In past works [23,24], the focus was mainly on the design of the optimum configuration of a single shielding coil. Here, a big improvement is proposed to optimize the configuration of several active coils to mitigate the field in a three-dimensional region by using optimization techniques. This new approach is described in the following sections. 


\section{Optimization and Design}

\subsection{Problem Definition}

Let us consider the $n$-coils active shielding configuration used to mitigate the magnetic field produced by a two-coil WPT system (i.e., $T x$ and $R x$ coils). The considered configuration can be modeled by $n+2$ equivalent circuits that are inductively coupled together, as shown in Figure 2 [23].

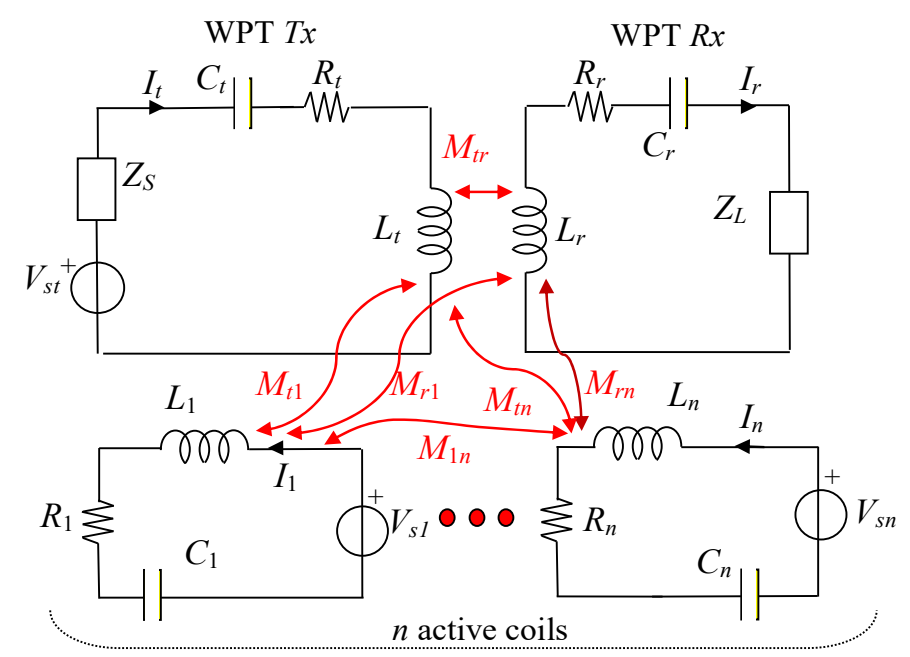

Figure 2. Equivalent circuit of the wireless power transfer (WPT) system with $n$ active shielding coils.

All the $n$ active coils are independently powered by voltage generators, as well as the $T x$ coil, while the $R x$ coil is not powered but only loaded on the impedance $Z_{L}$. Adopting circuital mesh equations, the system is given by:

$$
\left[\begin{array}{cc}
{\left[Z_{\text {wpt }}\right]} & {\left[Z_{\text {wpt }, a}\right]} \\
{\left[Z_{a, \text { wpt }}\right]} & {\left[Z_{a}\right]}
\end{array}\right]\left[\begin{array}{c}
{\left[I_{\text {wpt }}\right]} \\
{\left[I_{a}\right]}
\end{array}\right]=\left[\begin{array}{c}
{\left[V_{\text {wpt }}\right]} \\
{\left[V_{a}\right]}
\end{array}\right]
$$

where $\left[Z_{\text {wpt }}\right]$ is the $2 \times 2$ mesh impedance matrix of the WPT system composed by $T x$ and $R x$ coils given by:

$$
\left[Z_{w p t}\right]=\left[\begin{array}{cc}
Z_{t}+Z_{S} & Z_{t r} \\
Z_{t r} & Z_{r}+Z_{L}
\end{array}\right]
$$

being $Z_{S}$ the internal impedance of the $T x$ voltage source, $Z_{L}$ the load impedance of the $R x$ coil, $Z_{r}$ and $Z_{t}$ the impedances of the $R x$ and $T x$ compensated coils, respectively, and $Z_{t r}$ the mutual impedance between them. These last impedances are defined in the following.

The term $\left[Z_{a}\right]$ in (1) is the $n \times n$ mesh impedance matrix of the $n$ active coils given by:

$$
\left[Z_{a}\right]=\left[\begin{array}{ccc}
Z_{11} & \cdots & Z_{1 n} \\
\vdots & \ddots & \vdots \\
Z_{1 n} & \cdots & Z_{n n}
\end{array}\right]
$$

and $\left[Z_{a, w p t}\right]=\left[Z_{w p t, a}\right]^{T}$ is the $n \times 2$ mesh impedance matrix representing the interaction between the two WPT coils and the $n$ active coils given by:

$$
\left[Z_{a, w p t}\right]=\left[\begin{array}{cc}
Z_{t 1} & Z_{r 1} \\
\vdots & \vdots \\
Z_{t n} & Z_{r n}
\end{array}\right]=\left[Z_{w p t, a}\right]^{T}
$$


In (2)-(4), the subscripts $t$ and $r$ refer to the $T x$ and the $R x$ coils, respectively, and the subscripts 1 , $\ldots, n$ refer to the active coils. The coefficients of the impedance matrices are defined as:

$$
Z_{k l}= \begin{cases}R_{k}+j \omega L_{k}+\frac{1}{j \omega C_{k}}, & k=l, k \in\{t, r, 1, \ldots, n\} \\ j \omega M_{k l}, & k \neq l, k, l \in\{t, r, 1, \ldots, n\}\end{cases}
$$

where $R_{k}$ and $L_{k}$ are series resistance and inductance of the $k$ th coil, $M_{k l}$ is mutual inductance between the $k$ th and $l$ th coils, and $C_{k}$ is the series capacitance of the $k$ th coil. The resonance capacitances $C_{k}$ are chosen such that $L_{k} C_{k}=1 / \omega^{2}$ being $\omega=2 \pi f$ and $f$ the operational frequency equal to $85 \mathrm{kHz}$ in the considered automotive application.

Assuming the same notation described above, the current vectors in (1) are given by:

$$
\begin{gathered}
{\left[I_{\text {wpt }}\right]=\left[\begin{array}{c}
I_{t} \\
I_{r}
\end{array}\right]} \\
{\left[I_{a}\right]=\left[\begin{array}{c}
I_{1} \\
\vdots \\
I_{n}
\end{array}\right]}
\end{gathered}
$$

while the voltage source vectors in (1) are given by:

$$
\begin{gathered}
{\left[V_{\text {wot }}\right]=\left[\begin{array}{c}
V_{s t} \\
0
\end{array}\right]} \\
{\left[V_{a}\right]=\left[\begin{array}{c}
V_{s 1} \\
\vdots \\
V_{s n}
\end{array}\right]}
\end{gathered}
$$

where $V_{s t}$ is the known voltage generator on the $T x$ side, while the generic coefficient $V_{s i}$ of $\left[V_{a}\right]$ is the excitation on the $i$ th active coil that must be optimized.

\subsection{Optimization Algorithm}

First of all, the geometrical configuration of the WPT and active coils must be a priori defined. Then, the magnetic flux density $\mathbf{B}(P)=B_{x}(P) \widehat{\mathbf{x}}+B_{y}(P) \widehat{\mathbf{y}}+B_{z}(P) \widehat{\mathbf{z}}$ generated by all coil currents at point $P=(x, y, z)$ is given by superposition when assuming linearity as:

$$
\mathbf{B}(P)=\sum_{k} \mathbf{b}_{k}(P) I_{k}=\left[\begin{array}{lllll}
\mathbf{b}_{t}(P) & \mathbf{b}_{r}(P) & \mathbf{b}_{1}(P) & \cdots & \mathbf{b}_{n}(P)
\end{array}\right]\left[\begin{array}{c}
{\left[I_{w p t}\right.} \\
{\left[I_{a}\right]}
\end{array}\right]
$$

where

$$
\mathbf{b}_{k}(P)=b_{k, x}(P) \widehat{\mathbf{x}}+b_{k, y}(P) \widehat{\mathbf{y}}+b_{k, z}(P) \hat{\mathbf{z}}=\left[\begin{array}{lll}
\widehat{\mathbf{x}} & \widehat{\mathbf{y}} & \widehat{\mathbf{z}}
\end{array}\right]\left[\begin{array}{c}
b_{k, x}(P) \\
b_{k, x}(P) \\
b_{k, x}(P)
\end{array}\right]
$$

is the magnetic field vector transfer function generated by the $k$ th coil unit current (measure unit: T/A) [20]. By introducing (11) into (10), the magnetic flux density at point $(P)$ is rewritten as:

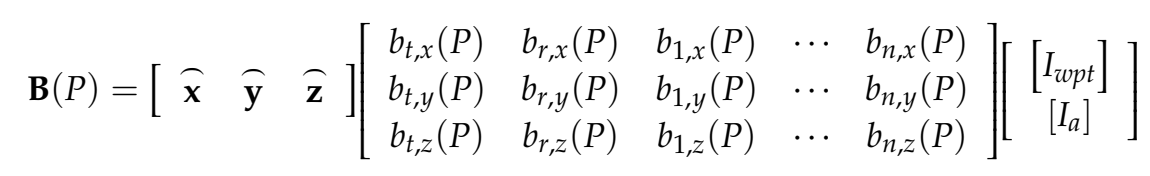


or, in a more compact form, as:

$$
\mathbf{B}(P)=\left[\begin{array}{lll}
\widehat{\mathbf{x}} & \widehat{\mathbf{y}} & \widehat{\mathbf{z}}
\end{array}\right][b(P)][I]
$$

where $[b(P)]$ is the $3 \times(n+2)$ matrix where the $k$ th column is given by the three components of $b_{k}(P)$ as in (11), and $[I]=\left[\left[I_{\text {wpt }}\right]\left[I_{a}\right]\right]^{T}$ is the current vector. According to (13), the $3 \times 1$ vector $[B(P)]$ having for coefficients the three components of the magnetic flux density at point $P$ is given by:

$$
[B(P)]=\left[\begin{array}{c}
B_{x}(P) \\
B_{y}(P) \\
B_{z}(P)
\end{array}\right]=[b(P)][I]
$$

The interest is focused on minimizing the magnetic field in critical zones accessible to humans such as in Region 2 beside the EV. To this purpose, we consider this region as a cloud of $m$ points beside the EV close to the ground, as shown in Figure 3. At each point $P_{j}=\left(x_{j}, y_{j}, z_{j}\right)$ with $j=1, \ldots, m$ of the cloud, the transfer functions $\mathbf{b}_{\boldsymbol{k}}(P)$ given by (11) is calculated for all coils, i.e., $k \in\{t, r, 1, \ldots, n\}$, assuming a unit current excitation $I_{k}=1 \mathrm{~A}$. Then, the Gradient Descent [25] optimization technique is used to find out the values of the active coils currents which minimize the magnetic field in the cloud of points as described in the following.

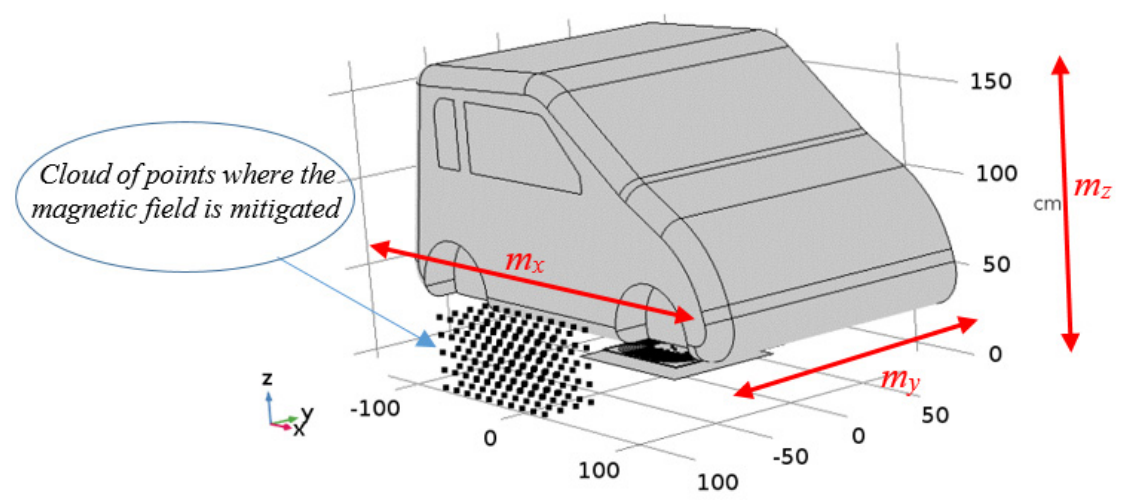

Figure 3. Cloud of points beside the EV where the magnetic field is mitigated.

By writing (14) for all the $m$ points of the cloud, it is possible to obtain the vector:

$$
\left[\begin{array}{c}
{\left[B\left(P_{1}\right)\right]} \\
{\left[B\left(P_{2}\right)\right]} \\
\vdots \\
{\left[B\left(P_{m}\right)\right]}
\end{array}\right]=[S]\left[\begin{array}{c}
{\left[I_{w p t}\right]} \\
{\left[I_{a}\right]}
\end{array}\right]
$$

where the matrix $[S]$ of size $3 m \times(n+2)$ is given by:

$$
[S]=\left[\begin{array}{c}
{\left[b\left(P_{1}\right)\right]} \\
\vdots \\
{\left[b\left(P_{m}\right)\right]}
\end{array}\right]
$$

By deriving the current vector from (1) and introducing it in (15), it yields:

$$
\left[\begin{array}{c}
{\left[B\left(P_{1}\right)\right]} \\
{\left[B\left(P_{2}\right)\right]} \\
\vdots \\
{\left[B\left(P_{m}\right)\right]}
\end{array}\right]=[S]\left[\begin{array}{cc}
{\left[Z_{w p t}\right]} & {\left[Z_{\text {wpt }, a}\right]} \\
{\left[Z_{a, w p t}\right.} & {\left[Z_{a}\right]}
\end{array}\right]^{-1}\left[\begin{array}{c}
{\left[V_{w p t}\right]} \\
{\left[V_{a}\right]}
\end{array}\right]=[G]\left[\begin{array}{c}
{\left[V_{w p t}\right]} \\
{\left[V_{a}\right]}
\end{array}\right]
$$


where for compactness it has used the position:

$$
[G]=[S]\left[\begin{array}{cc}
{\left[Z_{w p t}\right]} & {\left[Z_{w p t, a}\right]} \\
{\left[Z_{a, w p t}\right]} & {\left[Z_{a}\right]}
\end{array}\right]^{-1}
$$

To determine the active coils excitation, i.e., $\left[V_{a}\right]$, it is useful to rearrange (17) as follows:

$$
\left[\begin{array}{c}
{\left[B\left(P_{1}\right)\right]} \\
{\left[B\left(P_{2}\right)\right]} \\
\vdots \\
{\left[B\left(P_{m}\right)\right]}
\end{array}\right]=[A]\left[V_{a}\right]-[U]
$$

where $[A]$ is the $3 m \times n$ submatrix of $[G]$ obtained removing the first two columns of $[G]$, and $[U]$ is a column vector whose components are obtained by multiplying the $T x$ excitation $V_{s t}$ with the elements on the first column with a minus sign of $[G]$, as:

$$
\begin{gathered}
A_{j, h}=G_{j, h+2}, j \in\{1, \ldots, 3 m\}, h \in\{1, \ldots, n\} \\
U_{j}=-G_{j, 1} V_{s t}, j \in\{1, \ldots, 3 m\}
\end{gathered}
$$

To address the optimization problem, it is necessary to define the variables, the constraints, and the cost function. The variables are the coefficients of the voltage source vector $\left[V_{a}\right]$, while the constraints are all other physical and geometrical quantities which are kept fixed at a predetermined value. As the interest is here focused to minimize the norm of the magnetic flux density $\|\mathbf{B}\|^{2}$, considering (19), the cost function $J\left(\left[V_{a}\right]\right)$ is given by:

$$
J\left(\left[V_{a}\right]\right)=\frac{1}{2 m}\left([A]\left[V_{a}\right]-[U]\right)^{*}\left([A]\left[V_{a}\right]-[U]\right)
$$

where the superscript * represents the transpose complex conjugate. Equation (22) is proportional to the average value of $\|\mathbf{B}\|^{2}$ in the considered $m$ points. The update equation after the $k$ th iteration of the Gradient Descent is given by:

$$
\left[V_{a}\right]^{k+1}=\left[V_{a}\right]^{k}-\alpha \frac{1}{m}[A]^{*}\left([A]\left[V_{a}\right]^{k}-[U]\right)
$$

being $\alpha$ the learning rate $(0<\alpha<1)$ and where all coefficients of matrix $\left[V_{a}\right]$ are initially set to zero. After a sufficient number of iterations in (23), $\left[V_{a}\right]$ will converge and return the optimized value of the voltage generator of each active coil.

\section{Application}

The optimization procedure previously described is applied to design the multiple coils active shield for the wireless charging system of the EV. The considered configuration including active coils and WPT coils is shown in Figure 4, where the EV bodyshell is not depicted to make the system easily visible. The WPT coils are designed following the standard SAE J2954 $[7,26]$. The Tx coil, placed on the ground, has a circular shape with dimensions $x_{T x}=650 \mathrm{~mm}$ and $y_{T x}=500 \mathrm{~mm}$. The coil is composed by two parallel windings forming $N_{1}=8$ turns (the total equivalent turns are then $2 \times 8=16$ ). A Litz wire with an external diameter $\varnothing=5 \mathrm{~mm}$ is adopted for the coil. The $R x$ coil has a circular shape with a side length $s_{R x}=s_{r y}=380 \mathrm{~mm}$, it is composed by $N_{2}=10$ turns, and it is made with the same Litz wire of the $R x$ coil. On both the $T x$ and $R x$ coils, one magnetic (ferrite) and one conductive (aluminum) shields are included as described in the SAE standard [7]. The operational frequency is set to $f=85 \mathrm{kHz}$ while the power delivered to the vehicle battery is set to $7.7 \mathrm{~kW}$. The bodyshell of the considered EV (please see again Figure 3) has outer dimensions $m_{x}=274 \mathrm{~cm}, m_{y}=160 \mathrm{~cm}, m_{z}=140 \mathrm{~cm}$ [26]. The WPT receiving 
coil is placed in the center of the vehicle underbody. The bodyshell is simplified as a metallic box made of a high strength aluminum alloy with electrical conductivity $\sigma_{a l}=10 \mathrm{MS} / \mathrm{m}$ and thickness $t_{k}=2 \mathrm{~mm}$. To reproduce the worst-case scenario a very small vehicle is considered, since the distance between the coil and the people outside the vehicle is minimum. The vertical separation distance between the $T x$ and $R x$ coils is set to $d_{c}=25 \mathrm{~cm}$ to reproduce the worst case condition (the magnetic flux leakage increases with the gap between the $T x$ and $R x$ coils).

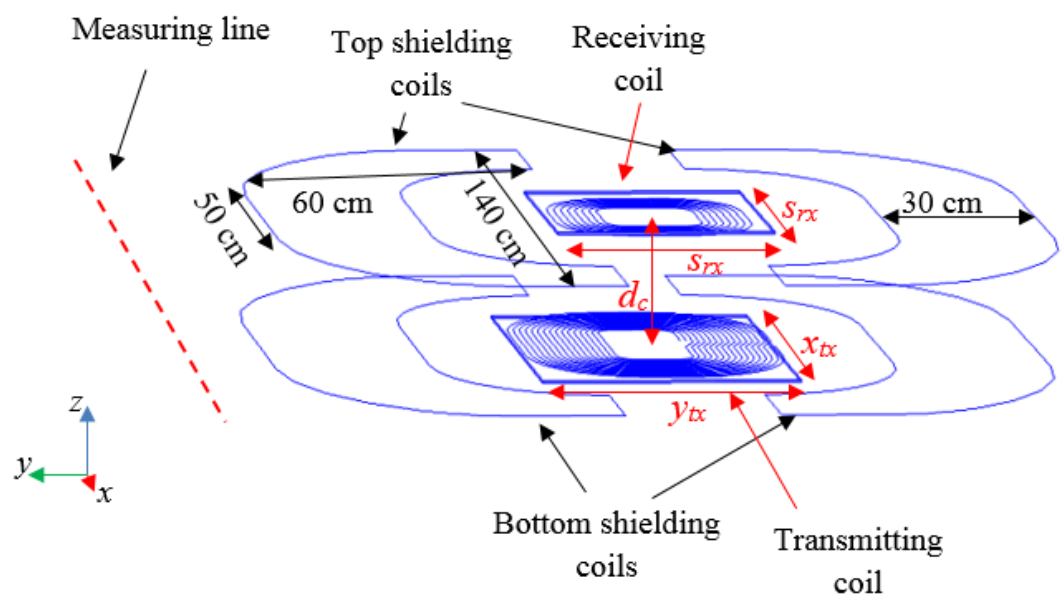

Figure 4. WPT and shielding coil configuration.

Four active coils are used to mitigate the magnetic field generated by the WPT coils: two on the transmitting side and two on the receiving side (see again Figure 4). The active coils are geometrically designed following the guidelines described in [24] to obtain good shielding capacity without degrading the WPT performances.

The computational domain is analyzed by the commercial software tool COMSOL [27] based on the finite element method (FEM) solution of the magneto-quasi-static (MQS) field equations. To reduce the computational cost, transition boundary conditions (TBCs) are used to model the metallic surfaces [27]. The TBCs take into account the field discontinuity, i.e., reflection and refraction, produced by thin conductive materials as the EV bodyshell or shields without any FEM discretization of these materials. It greatly reduces the number of unknowns and the solution time. In the proposed application, the FEM mesh is composed by 258547 2nd order tetrahedral elements and the degrees of freedom of equations were 1906778. The solution time using a PC with Intel Core i7 7 th gen CPU and 16 GB of RAM was 15 min and $42 \mathrm{~s}$. The COMSOL model of the proposed system is reported in Figures 5 and 6.

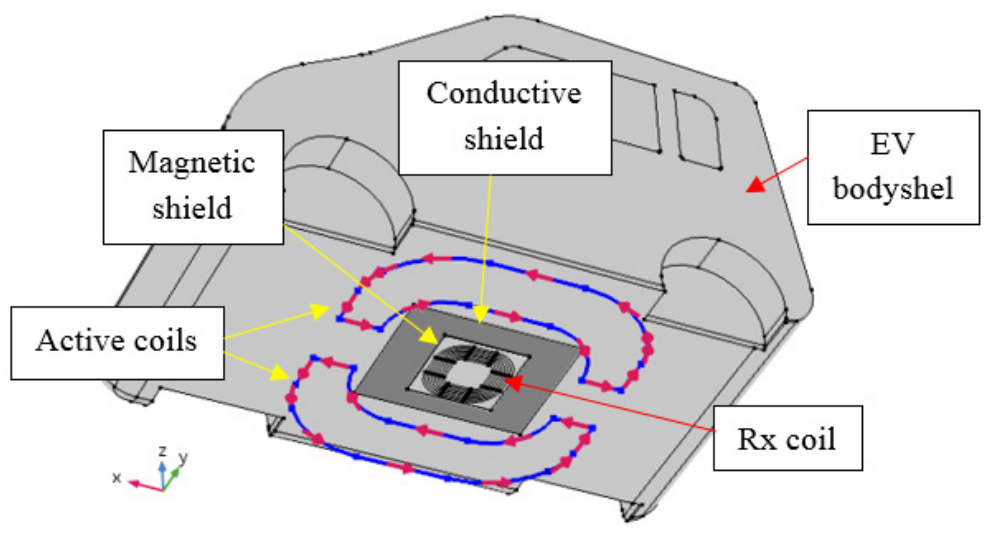

Figure 5. COMSOL model of the receiving coil $(R x)$ side of the system mounted on EV underbody. 


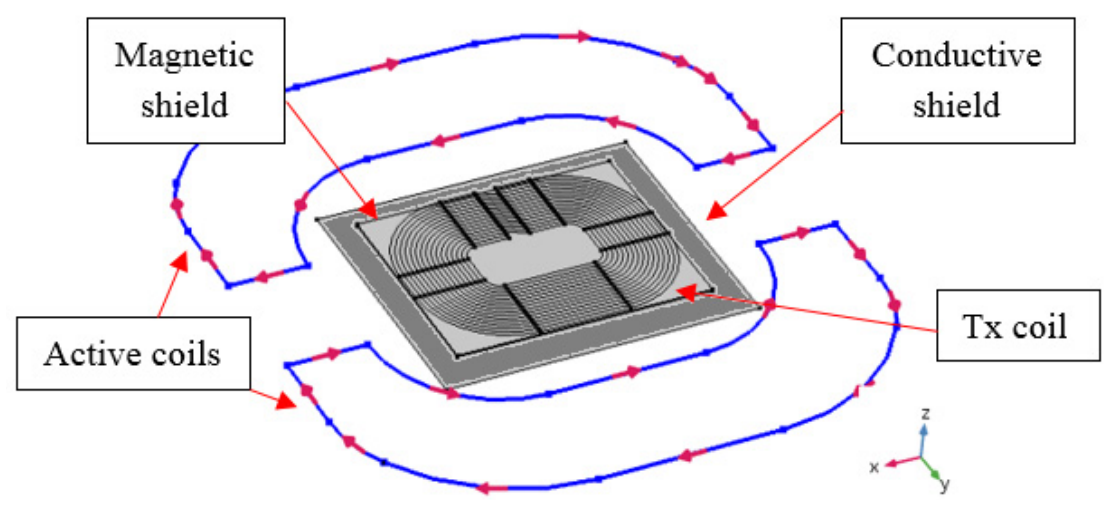

Figure 6. COMSOL model of the transmitting coil ( $T x)$ side of the system.

First, the magnetic field distribution produced by the WPT coil currents is calculated without considering the presence of the active coils. Then, two different active coil configurations are examined. In test case \#1, two active coils, named bottom coils, placed on the ground (see again Figure 4) are considered. In test case \#2, four active coils (the two previously described bottom coils on the ground, with additional two top coils placed in the EV underbody) are considered. The shape, position, and dimension of all active planar coils are prefixed, and the optimization procedure is carried out to obtain the active coil excitations which allow minimizing the magnetic field emission in the region beside the EV. To this aim, a total of $m=16,384$ points were selected, uniformly placed in two zones where the magnetic field must be mitigated and symmetrically located on the left and right sides of the vehicle, respectively. Each zone discretized in a cloud of points is beside the EV close to the ground, as shown schematically in Figure 3 for the right side.

The volume with the cloud of points is defined as $\left\{(x, y, z) \in \mathbb{R}^{3} \mid x_{1} \leq x \leq x_{2}, y_{1} \leq y \leq y_{2}, z_{1} \leq z \leq z_{2}\right\}$ assuming $x_{1}=-50 \mathrm{~cm}, x_{2}=50 \mathrm{~cm}, y_{1}=$ $85 \mathrm{~cm}, y_{2}=125 \mathrm{~cm}, z_{1}=0 \mathrm{~cm}$, and $z_{2}=50 \mathrm{~cm}$ for the right side zone, while $y_{1}=-125 \mathrm{~cm}$ and $y_{2}=-85$ $\mathrm{cm}$ on the left side zone.

For each of the considered $m$ points the vector transfer function $\mathbf{b}$ in (11) is calculated by COMSOL assuming a unit current in turn in each of the $2+n=2+4=6$ considered coils. Then, the optimization procedure based on the Gradient Descent algorithm is carried out by MATLAB to calculate the independent voltage sources of the active coils for the two test cases. The cost function $J\left(\left[V_{a}\right]\right)$ vs. iteration step for test case \#2 is shown in Figure 7.

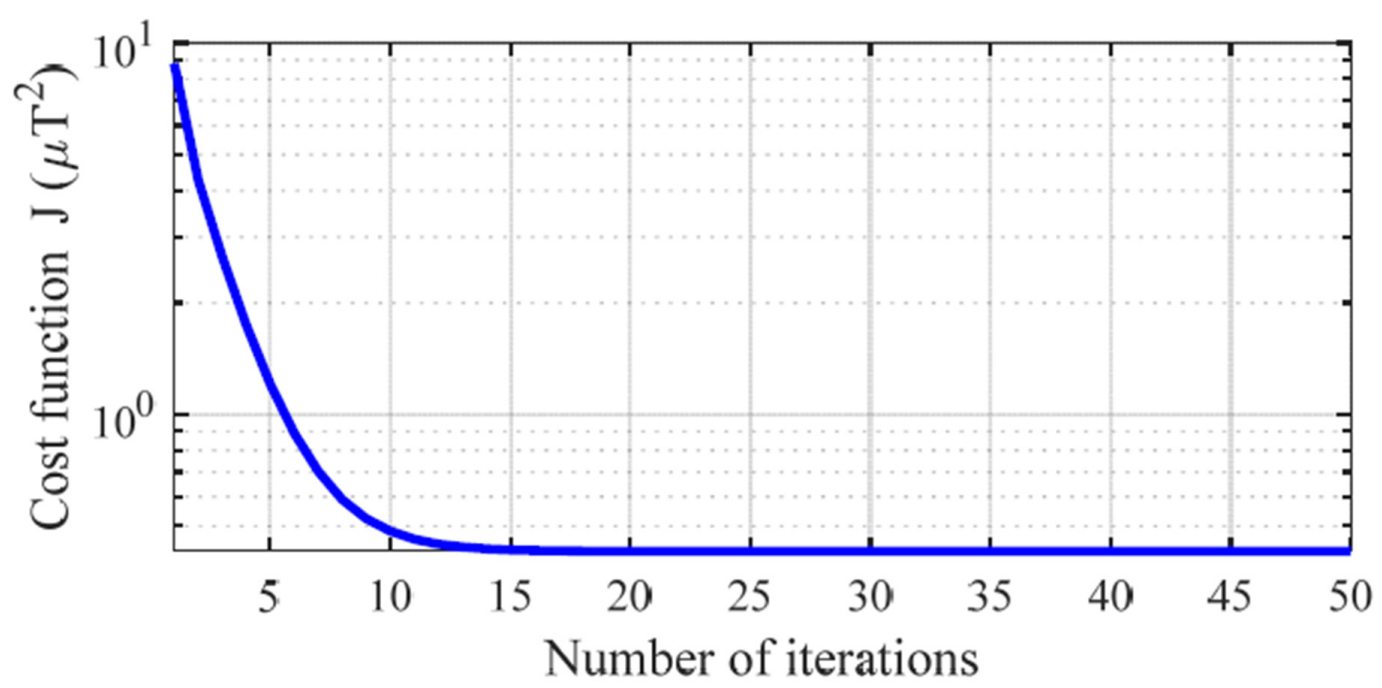

Figure 7. Cost function J vs. number of iterations with Gradient Descent for test case \#2. 
The ratio between the average of $\left\|\mathbf{B}_{\text {active }}\right\|$ calculated over all $m$ points used for the optimization and that of the incident field $\left\|\mathbf{B}_{\text {wpt }}\right\|$ (i.e., case without active coil shielding) versus the number of iterations used in the optimization is shown in Figure 8, for test case \#2. The lower this ratio is, the better the magnetic field shielding will be. Note that Figures 7 and 8 have similar decreasing behaviors until convergence.

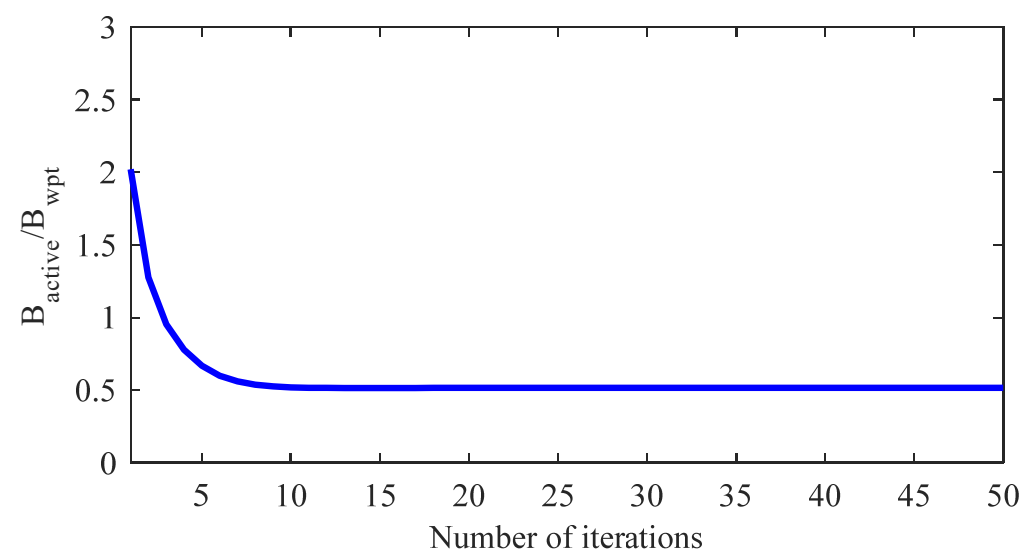

Figure 8. Ratio of $B_{\text {active }} / B_{\text {wpt }}$ vs. number of iterations of the Gradient Descent for test case \#2.

The optimization procedure provides the vector $\left[V_{a}\right]$ containing the voltage source excitations of the active coils. Applying these excitations to the circuit shown in Figure 2 for the two considered test cases \#1 and \#2, the coil currents are obtained. Thus, it is possible to impose these currents as sources of the magnetic field and to calculate the field distribution by COMSOL using the MQS equations solver. The magnetic field beside the EV is calculated along the red dotted measuring line shown in Figure 4. The comparison of the results obtained in test cases \#1 and \#2 is shown in Figure 9. The measuring line is located beside the EV parallel to the $x$ axis, at a distance of $110 \mathrm{~cm}$ from the center of the coil along the $y$ axis and equidistant from the primary and secondary coil on the $z$ axis. It is worth noting that the magnetic field is significantly reduced in both test cases \#1 and \#2, and the field mitigation is also very similar. Therefore, the configuration of test case \#1 is more convenient as it is based on only two active coils in the ground assembly instead of the four coils in test case \#2. A smaller number of active coils leads to a reduction in complexity, cost, and losses.

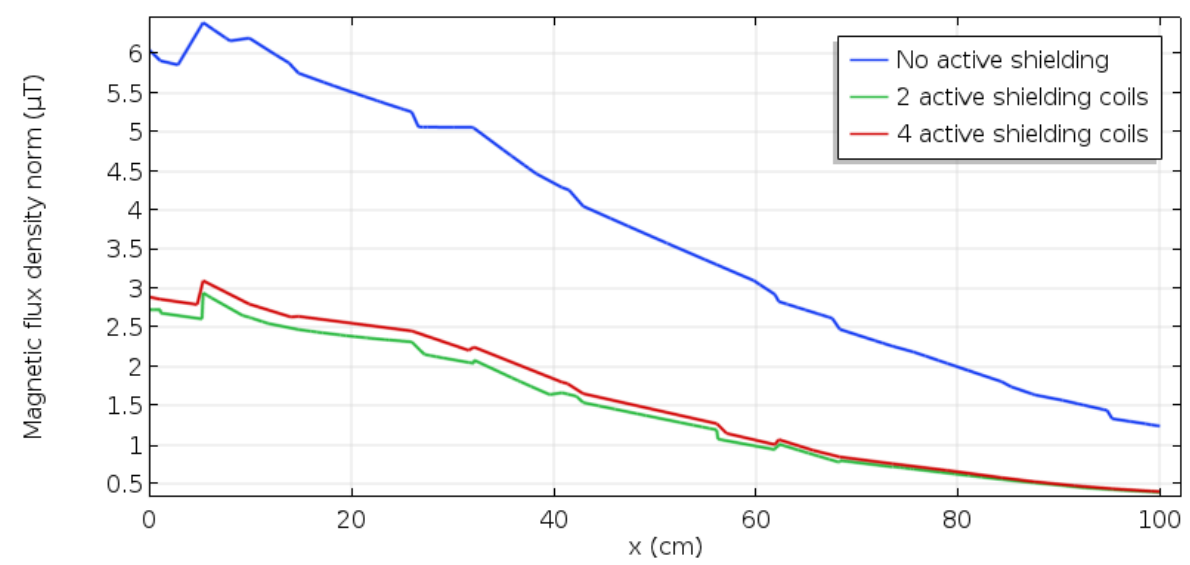

Figure 9. Magnetic field B along the measuring line beside the car with and without active shielding.

Then, we have investigated the relevance of the sampling (i.e., cloud of $m$ points) on the obtained results. Figure 10 shows the ratio of average $\|\mathbf{B}\|$ with and without active shielding versus the number $m$ of points used in the optimization. The points are taken in order of distance from the primary coil 
center (the closer ones before the further ones). For the considered configuration $m=16384$ points were used but, as you can see, the procedure can be considered accurate selecting approximately 1000 points.

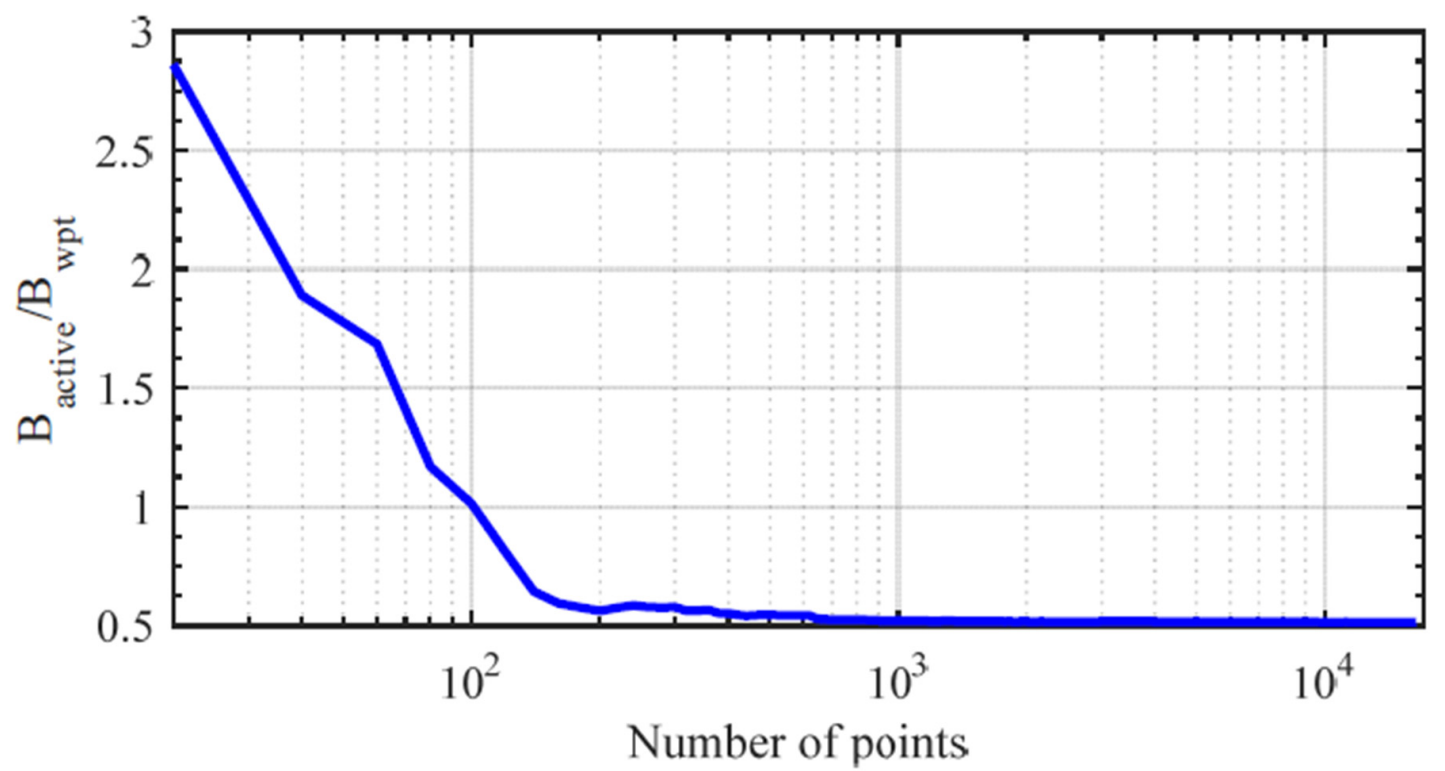

Figure 10. Ratio $B_{\text {active }} / B_{\text {wpt }}$ vs. number of points.

Finally, we observed as the WPT efficiency is strongly influenced by the quality of the applied active shielding, as shown in Figure 11. It can be noted that the optimization of the shielding coil current leads to a very limited reduction of the system efficiency. The proposed method can be applied to several configurations of the system (e.g., different gap, coil misalignment, etc.). For each condition, the optimum shielding current can be considered to create a lookup table in order to minimize the magnetic field for all operational conditions of the system.

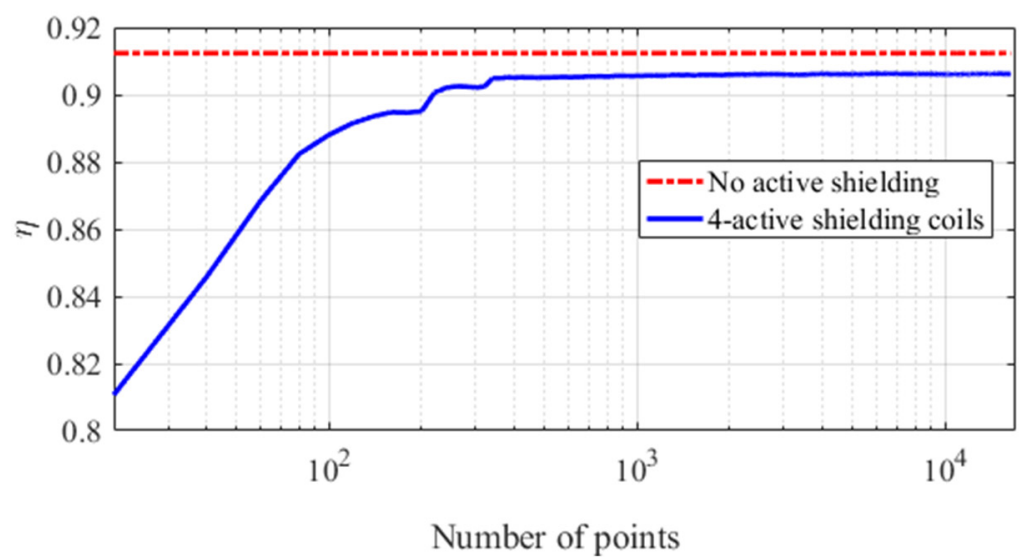

Figure 11. Efficiency $\eta$ vs. number of points.

\section{Conclusions}

An optimization procedure for the minimization of the magnetic field produced by a near field WPT system by active shielding has been presented. The proposed method permits finding the optimum current for each shielding active coil to minimize the magnetic field in a specific area while maintaining very good electrical efficiency. This aspect is very important for high power WPT systems. An automotive WPT charging system designed following the SAE standard has been considered to apply the proposed optimization procedure. The obtained results demonstrate that the magnetic field on the most critical area outside the EV, the magnetic field level, can be halved with only a 
little reduction of the WPT system efficiency, taking into account also the losses in the active coils. The efficiency goes from $91.2 \%$ without the shielding active WPT to $90.6 \%$ with the active shielding. Finally, it should be noted that the proposed method can be applied to different electro-geometrical configurations of active and WPT coils.

Author Contributions: S.C., T.C., F.M. and M.F. conceived and planned the simulations. S.C. and F.M. developed the algorithms. T.C. and M.F. realize the simulations set-up. All authors provided critical feedback, improved the final design, analyzed the data and wrote the paper. All authors have read and agreed to the published version of the manuscript.

Funding: This research was financed by research projects of University of L'Aquila and Sapienza University of Rome.

Conflicts of Interest: The authors declare no conflict of interest.

\section{References}

1. Covic, G.A.; Boys, J.T. Inductive power transfer. Proc. IEEE 2013, 101, 1276-1289. [CrossRef]

2. Shinohara, N. Power without wires. IEEE Microw. Mag. 2011, 11, 64-73. [CrossRef]

3. Wang, C.-S.; Covic, G.A.; Stielau, O.H. Power Transfer Capability and Bifurcation Phenomena of Loosely Coupled Inductive Power Transfer Systems. Trans. Ind. Electron. 2004, 51, 148-157. [CrossRef]

4. Ahmad, A.; Alam, M.S.; Chabaan, R.A. Comprehensive Review of Wireless Charging Technologies for Electric Vehicles. Trans. Transp. Electrif. 2018, 4, 38-63. [CrossRef]

5. ISO/PAS 19363:2017. Electrically Propelled Road Vehicles. Connection to an External Electric Power Supply. Safety Requirements. Available online: http://dx.doi.org/10.3403/30321062u (accessed on 18 April 2019).

6. IEC 61980-1:2015. Electric Vehicle Wireless Power Transfer (WPT) Systems-Part 1: General Requirements. Available online: https://webstore.iec.ch/publication/22951 (accessed on 18 April 2019).

7. SAE Recommended Practice J2954 (rev. 201711). Wireless Power Transfer for Light-Duty Plug-In/Electric Vehicles and Alignment Methodology. SAE Int. 2017. [CrossRef]

8. Campi, T.; Cruciani, S.; Feliziani, M. Numerical characterization of the magnetic field in electric vehicles equipped with a WPT system. Wirel. Power Transf. 2017, 4, 78-87. [CrossRef]

9. Campi, T.; Cruciani, S.; Feliziani, M. Wireless power transfer (WPT) system for an electric vehicle (EV): How to shield the car from the magnetic field generated by two planar coils. Wirel. Power Transf. 2017, 5, 1-8. [CrossRef]

10. De Santis, V.; Campi, T.; Cruciani, S.; Laakso, I.; Feliziani, M. Assessment of the Induced Electric Fields in a Carbon-Fiber Electrical Vehicle Equipped with a Wireless Power Transfer System. Energies 2018, 11, 684. [CrossRef]

11. Ding, P.; Bernard, L.; Pichon, L. Evaluation of Electromagnetic Field in Human Body Exposed to Wireless Inductive Charging System. IEEE Trans. Magn. 2014, 50, 1037-1040. [CrossRef]

12. Laakso, I.; Hirata, A. Evaluation of the induced electric field and compliance procedure for a wireless power transfer system in an electrical vehicle. Phys. Med. Biol. 2013, 58, 7583. [CrossRef] [PubMed]

13. Kim, H.; Song, C.; Kim, D.H.; Jung, D.H.; Kim, I.M.; Kim, Y.I.; Kim, J.; Ahn, S.; Kim, J. Coil design and measurements of automotive magnetic resonant wireless charging system for high-efficiency and low magnetic field leakage. IEEE Trans. Microw. Theory Tech. 2016, 64, 383-400. [CrossRef]

14. Campi, T.; Cruciani, S.; Feliziani, M. Magnetic Shielding of Wireless Power Transfer Systems. In Proceedings of the International Symposium on Electromagnetic Compatibility, Tokyo, Japan, 13-16 May 2014.

15. Mohammad, M.; Wodajo, E.T.; Choi, S.; Elbuluk, M.E. Modeling and Design of Passive Shield to Limit EMF Emission and to Minimize Shield Loss in Unipolar Wireless Charging System for EV. IEEE Trans. Power Electron. 2019, 34, 12235-12245. [CrossRef]

16. Kim, S.; Park, H.H.; Kim, J.; Kim, J.; Ahn, S. Design and analysis of a resonant reactive shield for a wireless power electric vehicle. IEEE Trans. Microw. Theory Tech. 2014, 62, 1057-1066. [CrossRef]

17. Moon, H.; Kim, S.; Park, H.H.; Ahn, S. Design of a resonant reactive shield with double coils and a phase shifter for wireless charging of electric vehicles. IEEE Trans. Magn. 2015, 51, 1-4. [CrossRef]

18. Park, J.; Kim, D.; Hwang, K.; Park, H.H.; Kwak, S.I.; Kwon, J.H.; Ahn, S. A Resonant Reactive Shielding for Planar Wireless Power Transfer System in Smartphone Application. IEEE Trans. Electromagn. Compat. 2017, 59, 695-703. [CrossRef] 
19. Guidelines for limiting exposure to time-varying electric, magnetic, and electromagnetic fields (up to $300 \mathrm{GHz}$ ). International Commission on Non-Ionizing Radiation Protection. Health Phys. 1998, 74, 494-522. Available online: https://www.ncbi.nlm.nih.gov/pubmed/9525427 (accessed on 18 April 2019).

20. Guidelines for limiting exposure to time-varying electric and magnetic fields for low frequencies $(1 \mathrm{~Hz}-100 \mathrm{kHz})$. International Commission on Non-Ionizing Radiation Protection. Health Phys. 2010, 99, 818-836. Available online: https://www.ncbi.nlm.nih.gov/pubmed/21068601 (accessed on 18 April 2019).

21. International Commission on Non-Ionizing Radiation Protection. Guidelines for Limiting Exposure to Electromagnetic Fields (100 kHz to $300 \mathrm{GHz}$ ). Health Phys. 2020, 118, 483-524. [CrossRef] [PubMed]

22. Buccella, C.; Feliziani, M.; Fuina, V. ELF magnetic field mitigation by active shielding. In Proceedings of the IEEE International Symposium on Industrial Electronics, L'Aquila, Italy, 8-11 July 2002; pp. 994-998.

23. Campi, T.; Cruciani, S.; Maradei, F.; Feliziani, M. Active coil system for magnetic field reduction in an automotive wireless power transfer System. In Proceedings of the 2019 IEEE International Symposium on Electromagnetic Compatibility, Signal \& Power Integrity (EMC + SIPI), New Orleans, LA, USA, 22-29 July 2019.

24. Cruciani, S.; Campi, T.; Maradei, F.; Feliziani, M. Active shielding design for wireless power transfer systems. IEEE Trans. Electromagn. Compat. 2019, 61, 1953-1960. [CrossRef]

25. Bishop, C.M. Pattern Recognition and Machine Learning; Springer: New York, NY, USA, 2006.

26. Campi, T.; Cruciani, S.; Maradei, F.; Feliziani, M. Magnetic Field during Wireless Charging in an Electric Vehicle According to Standard SAE J2954. Energies 2019, 12, 1795. [CrossRef]

27. COMSOL Multiphysics. Available online: https://www.comsol.com/ (accessed on 23 October 2020).

Publisher's Note: MDPI stays neutral with regard to jurisdictional claims in published maps and institutional affiliations. 